\title{
Transcriptional Regulations on the Low-Temperature-Induced Floral Transition in an Orchidaceae Species, Dendrobium nobile: An Expressed Sequence Tags Analysis
}

\author{
Shan Liang, ${ }^{1}$ Qing-Sheng Ye, ${ }^{1}$ Rui-Hong Li, ${ }^{1}$ Jia-Yi Leng, ${ }^{1}$ Mei-Ru Li, ${ }^{2}$ \\ Xiao-Jing Wang, ${ }^{1}$ and Hong-Qing $\mathrm{Li}^{1}$ \\ ${ }^{1}$ Guangdong Provincial Key Laboratory of Biotechnology for Plant Development, School of Life Science, South China Normal University, \\ Guangzhou 510631, China \\ ${ }^{2}$ Key Laboratory of Plant Resources Conservation and Sustainable Utilization, South China Botanical Garden, \\ Chinese Academy of Sciences, Guangzhou 510650, China
}

Correspondence should be addressed to Xiao-Jing Wang, wangxj@scnu.edu.cn and Hong-Qing Li, hqli@scnu.edu.cn

Received 30 July 2011; Revised 21 December 2011; Accepted 8 January 2012

Academic Editor: G. Pesole

Copyright (C) 2012 Shan Liang et al. This is an open access article distributed under the Creative Commons Attribution License, which permits unrestricted use, distribution, and reproduction in any medium, provided the original work is properly cited.

\begin{abstract}
Vernalization-induced flowering is a cold-relevant adaptation in many species, but little is known about the genetic basis behind in Orchidaceae species. Here, we reported a collection of 15017 expressed sequence tags (ESTs) from the vernalized axillary buds of an Orchidaceae species, Dendrobium nobile, which were assembled for 9616 unique gene clusters. Functional enrichment analysis showed that genes in relation to the responses to stresses, especially in the form of low temperatures, and those involving in protein biosynthesis and chromatin assembly were significantly overrepresented during 40 days of vernalization. Additionally, a total of 59 putative flowering-relevant genes were recognized, including those homologous to known key players in vernalization pathways in temperate cereals or Arabidopsis, such as cereal VRN1, FT/VRN3, and Arabidopsis AGL19. Results from this study suggest that the networks regulating vernalization-induced floral transition are conserved, but just in a part, in $D$. nobile, temperate cereals, and Arabidopsis.
\end{abstract}

\section{Introduction}

Transition from the vegetative phase to the flowering phase is crucial to both development and reproduction in plants. Besides endogenous signals, this process is also affected by external cues such as day length and temperature. Vernalization, an exposure to low temperature extending over a period differing from species to species, is an adaptive nature to ensure some plants survival in harsh winters and flowers under a favourable condition in spring. In the dicot model Arabidopsis, vernalization-regulated flowering is mediated by both FLC-dependant and -independent pathways $[1,2]$, in which the gene VIN3 (which interacts with VRN2), a key upstream component, is probably activated by exposure to low temperature and subsequently leads to changes in histone methylation of downstream gene regions [3]. For example, the expression of FLC is suppressed by vernalization through enrichment of $\mathrm{H} 3 \mathrm{~K} 27 \mathrm{~m} 3$ on the chromatin [3], which consequently releases $F T$ and $S O C 1$ from inhibition by FLC to promote the transition to flowering. This FLC-dependant pathway is regulated by both temperature and day length [4]. AGL19, a close relative of $S O C 1$, is believed to mediate an $F L C$-independent pathway that activates flowering under vernalization in Arabidopsis [1]. This process is associated with a cold-induced decrease of H3K27m3 on AGL19 locus and probably also with the loss of function of CLF and MSI1 or the involved complex [5]. Ectopically expression of Arabidopsis AGL19 leads to only mild abnormalities, suggesting that AGL19 has a limited role in flowering control in Arabidopsis [1]. In addition to FLC and AGL19, MAF2 and AGL24 are also involved in the floral transition in Arabidopsis $[6,7]$. At present, $F L C$ is thought to be central to the control of flowering in Arabidopsis and probably also in most other dicots $[1,2]$. 
Monocots, however, are likely to be different: no monocot orthologs of Arabidopsis FLC have been found so far. In temperate cereals such as wheat and barley, responses to vernalization are mediated by VRN1, VRN2, and VRN3 [2]. Cereal VRN1 has two roles, namely, (a) to transmit the cold signal and thereby induce VRN3, the ortholog of AtFT in cereals [11] and (b) to act as a floral meristem identify which is activated by FT/VRN3 [12]. Thus, VRN1 and FT/VRN3 form a positive feedback loop and regulate each other under vernalization. VRN1 may also have a role in storing the memory of vernalization through methylation of Histone 3 [13], which resembles the epigenetic property of Arabidopsis FLC. However, the exact mechanism of this process remains unclear so far. Cereal VRN2 acts as a flowering repressor that is regulated by both low temperatures and day length [14]. Cold can lower the expression of VRN2 [15], thereby releasing FT/VRN3 from repression [16]. VRN2 may be inhibited by VRN1 under vernalization $[9,10,14]$. To summarize, vernalization-induced regulation of flowering in monocots is evolutionarily divergent from that in dicots, although the networks are similar to some extent [2].

Orchidaceae is the largest family in the plant kingdom and is considered particularly speciational, indicating that Orchidaceae species are well adapted to the surrounding environments. Dendrobium is a vast genus consisting of more than a thousand species that are native to South Asia, Australia, New Zealand, and Oceania [17]. Species of this genus differ in the extent of dependence on vernalization. For instance, Dendrobium phalaenopsis can flower at high temperatures whereas Dendrobium nobile requires vernalization and flowers only after a spell of exposure to relatively low temperatures [18]. D. nobile is used as a herbal medicine and is popular as a potted ornament prized by growers and hobbyists for its beautiful flowers. Its juvenile period lasts for at least 3-5 years, which makes it difficult to meet the commercial demands. To accelerate flowering, potted $D$. nobile plants are always kept at low temperatures in a temperature-controlled greenhouse or grown in naturally cool environments such as in high mountains. In the laboratory, low-temperature treatment has been adopted for shortening the juvenile phase and promoting flowering [19]. Plants exposed to a constant temperature of $13^{\circ} \mathrm{C}$ flowered early whereas those at $18^{\circ} \mathrm{C}$ remained vegetative and did not flower $[18,20]$. To summarize, vernalization can promote flowering in this species. However, the genetic basis of low-temperature-induced flowering in $D$. nobile remains poorly understood.

Although a number of genes have been identified in orchids $[21,22]$, and several datasets of expressed sequence tags (ESTs) from flowering buds of Orchidaceae species have been deposited in GenBank, only a few of these genes have been characterized. Examples come to DOMADS1 and DOH1 from Dendrobium Madame Thong-In which are believed to be involved in the floral transition [23, 24]. Genetic networks underlying the biological features of vernalization in orchids have not yet been established.

In the present study, we created a collection of ESTs from axillary buds of $D$. nobile plants that had been vernalized for different durations. Changes in gene categories over time during the course of vernalization were studied and a group of candidate genes involved in flowering were identified. Based on the EST analysis, we propose a preliminary genetic network for vernalization-induced flowering in $D$. nobile.

\section{Material and Methods}

2.1. Plant Material. Plants of D. nobile were grown without regulating the photoperiod in a greenhouse in School of Life Science at South China Normal University (China). Adult plants were selected for experimental use. Plants for the lowtemperature treatment were moved to an air-conditioned greenhouse with the temperature setting at $15^{\circ} \mathrm{C}$ during the day and $10^{\circ} \mathrm{C}$ at night. The temperature regime was maintained over a different number of days $(5,10,20$, and 30 days) and the plants were sampled immediately upon moving to the greenhouse $(0 \mathrm{~d})$ and after the set duration $(5 \mathrm{~d}, 10 \mathrm{~d}$, $20 \mathrm{~d}$, and $30 \mathrm{~d}$ ). Control plants were grown under natural conditions (the average temperature was $26.1^{\circ} \mathrm{C}$ ). A total of 5-7 axillary buds close to the shoot tip of each seedling were harvested at the same time of the day at each sampling to avoid the possible influence of circadian rhythms. Excepting the whole apex, some other subtending tissues including the vascular transition zone and immature leaf bases had to be retained due to the difficulties in sampling. Totally, 70 seedlings were sampled, and the buds from each treatment were pooled. All buds were frozen under liquid nitrogen and stored at $-80^{\circ} \mathrm{C}$ until required.

2.2. RNA Extraction and Purification. Total RNA was prepared from the axillary buds from each treatment and control sample. Total RNAs from two different samples were pooled as described in additional file 1 in Supplementary Material available online at doi: 10.1155/2012/757801 for library construction, except for the control one $(0 \mathrm{~d})$. RNA was extracted using the RNeasy Plant Mini Kit (QIAGEN, Germany) but replacing the Trizol-based extracting buffer with a CTAB-containing one (2\% CATB, 1\% PEG4000, $1.4 \mathrm{M}$ $\mathrm{NaCl}, 1 \mathrm{mM}$ Tris $\cdot \mathrm{HCl}$ and $20 \mathrm{mM}$ EDTA, $\mathrm{pH} 8.2 \sim 8.5)$.

2.3. Library Construction. Full-length cDNA libraries were constructed using the Creator SMART cDNA Library Construction Kit (Clontech, USA) according to the manufacturer's instructions. The recombinant pDNR-LIB with $D$. nobile cDNA insertions was transformed into E. coli DH5 $\alpha$ and spread on the LB argar containing chloramphenicol. Monoclones were recovered by random selection.

2.4. Sequencing. Plasmids were isolated using the alkaline lysis method and used as starting templates for the subsequent sequencing. Insertions were sequenced from the $5^{\prime}$-end primed by M13 forward-sequencing primer using the Applied Biosystems 3730 DNA Analyzer. Before largescale sequencing, hundreds of monoclones were selected and sequenced to assess the frequency of both unloaded clones and multiple clones.

2.5. EST Analysis. Sequences longer than $100 \mathrm{bp}$ (600 bp on average) were considered for later analysis. Base calling and 
quality assignment of individual bases were done through Phred $(Q=20)[25]$. All the clean ESTs were assembled using Phrap ( minimatch $=42$ and miniscore $=20$ ). The assembled sequences (including contigs and singlets) were submitted to BLASTX for comparison against the nonredundant protein database available at NCBI (http://www.ncbi.nlm.nih.gov/) and the Arabidopsis protein database (TAIR10 proteins) at TAIR (http://www.arabidopsis.org/). ESTs from a single library were assembled and underwent BLASTX searches independently. Unigenes from different libraries were considered identical if the best hits in BLASTX matched the same protein.

To confirm the transcriptional change of the identified ESTs, real-time qPCR was performed for candidate ESTs or full-length cDNAs believed to be involved in flowering. Total RNA from each treatment duration was used as starting templates. Primer pairs for each candidate are listed in additional file 2.

For protein comparison, MEGA3 [26] was used to align homologous sequences. Amino acid identities were shown as percentage of conserved amino acids by pair wise comparison of the candidate protein and its corresponding homolog.

2.6. Gene Ontology (GO) Annotation. Gene ontology annotations were assigned to each unigene of $D$. nobile according to the best hit in BLASTX against the Arabidopsis TAIR10 protein database. Classifications were performed on the GOSlim-plant ontology of biological process. The frequency of each GOSlim-plant term was calculated as follows: (the number of nonredundant mUnigenes annotated by this GOSlim-plant term/total nonredundant annotated mUnigenes) $\times 100$. If a unigene was annotated by several GOSlimplant terms, it was classified into each of these terms but counted only once for the total count of nonredundant unigenes. GO annotations and GOSlim-plant categories of Arabidopsis proteins were retrieved as references from the TAIR website (http://www.arabidopsis.org/).

Overrepresented GO categories were extracted with BiNGO 2.3 [27] using hypergeometric statistics test and Benjamini and Hochberg False Discovery Rate (FDR) correction. GO categories with a corrected $P$ value $<0.05$ were considered overrepresented based on comparisons with the whole annotation of Arabidopsis. All these comparisons were performed on the ontology of biological process.

2.7. Real-Time Quantitative PCR. Total RNA was extracted from axillary buds of $D$. nobile plants exposed to low temperatures for $0,5,10,20$, and 30 days. In most cases, buds from 3 to 5 adult plants were pooled before RNA extraction. The qPCR reactions were run in the ABI PRISM1 7300 (Applied Biosystems) using the following program: hot start at $95^{\circ} \mathrm{C}$ for 30 seconds followed by 40 cycles of 5 seconds at $95^{\circ} \mathrm{C}$ and 31 seconds at $62^{\circ} \mathrm{C}$. In each $20 \mu \mathrm{L}$ qPCR reaction, cDNA initially generated from 5 to $7.5 \mathrm{ng}$ total RNA was used as a template. The $\mathrm{qPCR}$ reaction also contained gene-specific primer pairs ( $250 \mathrm{nM}$ each) and $10 \mu \mathrm{L}$ SYBR Green Premixture (TaKaRa) and $0.4 \mu \mathrm{L}$ Rox (TaKaRa). A total of 9 genes were assayed and the 18sRNA gene was used as the endogenous control. The primers used are listed in additional file 2. The sample from the $0 \mathrm{~d}$ batch was used for calibrating the expression level of each treatment duration. Three technical replicates were used for each combination of genes and the duration of exposure (days). Sterile distilled water instead of the cDNA template was used as the negative control. All the data were analysed using the SDSv1.3.0 software (ABI) based on the $2^{-\Delta \Delta C t}$ method [28]. The RQ values representing the relative expression level of a given gene under different treatment durations were $\log 2$-transformed in later analyses.

\section{Results and Discussion}

3.1. EST Collecting and Sequencing from D. nobile Axillary $B u d s$. Low-temperature treatment (artificial vernalization) of $D$. nobile can shorten the vegetative stage and accelerate flowering [19]. When compared with nonvernalized control, 10 days of exposure to low temperature produced a bulge in the node which would develop into several flower buds later, indicating that the transition from the vegetative stage to the flowering stage occurs at that time (Figure 1(b)). Extended low-temperature treatment hastened the development process (Figures 1(c) and 1(d)) and eventually led to the formation of floral organs from the bulged meristem.

To monitor the transcriptional changes during the lowtemperature-induced floral transition, three libraries were constructed using axillary buds collected at the end of the five treatment durations $(0,5,10,20$, and $30 \mathrm{~d})$. The bud samples were pooled, following the scheme shown in additional file 1 , to increase the variation in transcripts from different libraries. Another cDNA library constructed previously from 40-day-vernalization buds [29] was also included in the present study (additional file 1). Thus, we obtained four cDNA libraries presenting transcripts from distinct stages of vernalized buds. These libraries made it possible to perform a combined analysis and to detect changes in transcript abundance over time during vernalization.

About 3800 clones from each cDNA library were selected for sequencing, and 15381 reliable reads were obtained from the four libraries (GenBank accession numbers HO189246HO204626). After removing the low-quality reads and vector contaminants, 15017 clean ESTs were left, divided as follows: 3391 from the $0 \mathrm{~d}$ library, 3369 from the $(5+10) \mathrm{d}$ library, 4112 from the $(20+30) \mathrm{d}$ library, and 4145 from the $40 \mathrm{~d}$ library (Table 1). The average length of EST was $650 \mathrm{bp}$, and most fell into the range of 400-800 bp (additional file 3). Reads from different libraries were assembled independently using Phrap, resulting in 2976, 2983, 3491, and 3648 nonredundant Unigenes (including aSinglets and aContigs in Table 1) from the four datasets, respectively. The average percentage of nonredundant EST was 87.3 (and ranged from 84.9 to 88.54 ), indicating a relatively low redundancy within each library.

The number of the aContigs was probably underestimated in the assembly step, as some pseudounassembled ESTs can match to the same protein in BlastX analysis. We placed such EST sequences into a contig manually and designated as a mContigs (additional file 7). An EST sequence that 


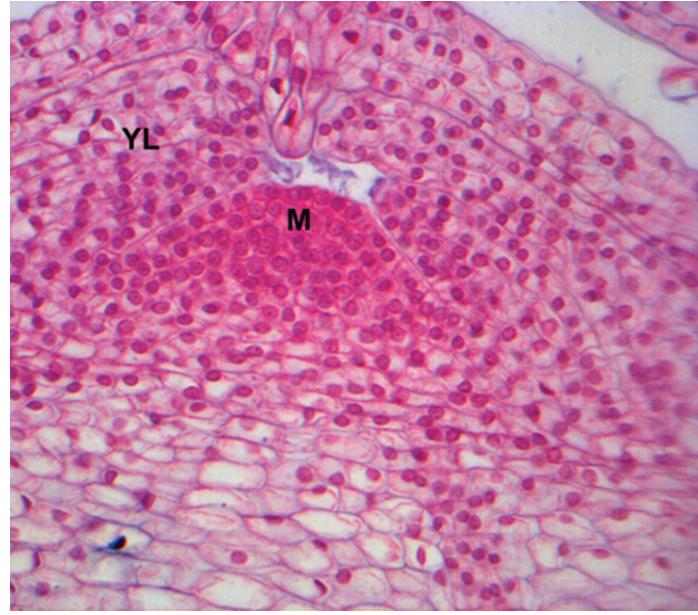

(a)

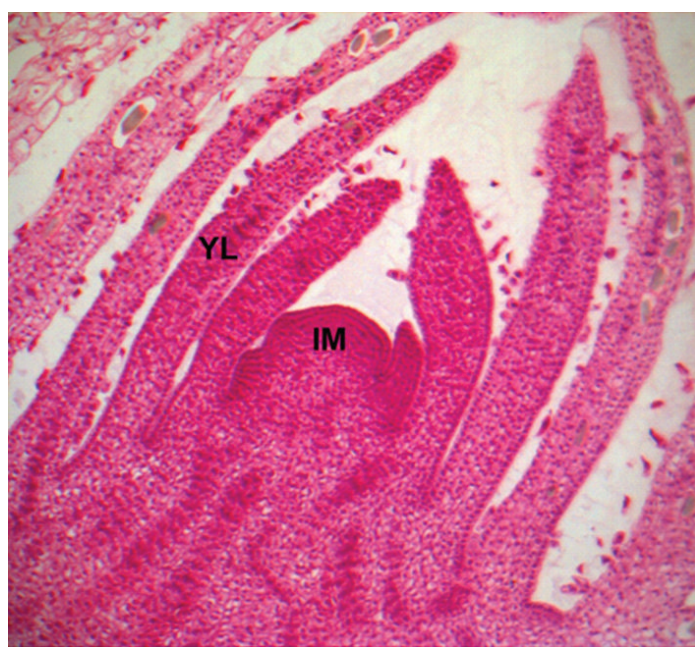

(c)

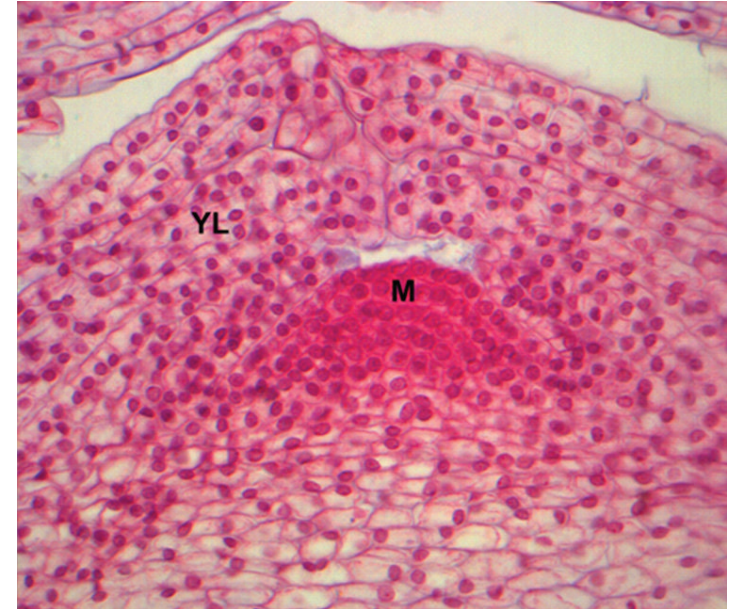

(b)

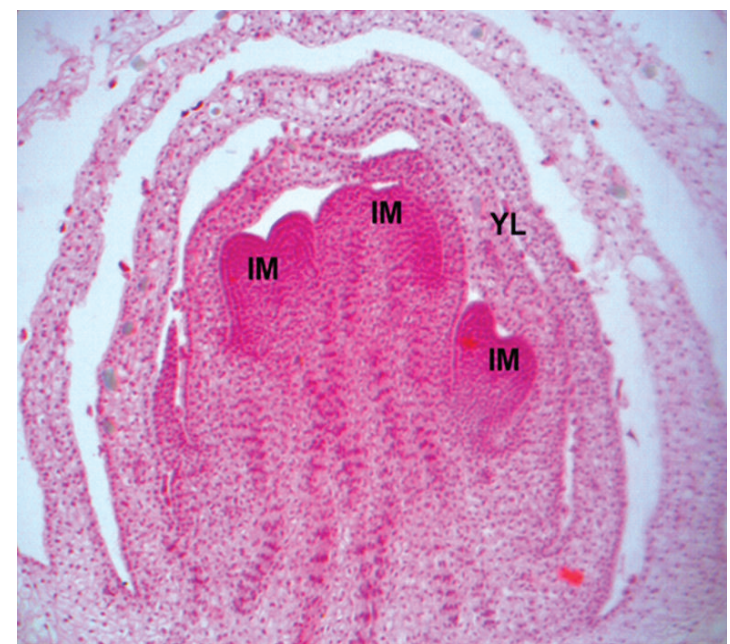

(d)

Figure 1: Low-temperature-induced anatomical changes in axillary buds of $D$. nobile. Axillary buds were sampled from seedlings of $D$. nobile, which were vernalized at $15 / 10^{\circ} \mathrm{C}$ (day/night) for (a) 0 , (b) 10, (c) 15, and (d) 30 days. The buds were first fixed by FAA and stained with Ehrlich's haematoxylin, and then embedded in paraffin to section serially. Anatomical examinations were carried out under microscope with magnification of $40 \times 10(\mathrm{a}, \mathrm{b})$ and $4 \times 10(\mathrm{c}, \mathrm{d})$. Visible changes could be found on meristem tissues of axillary buds after lowtemperature exposure, from bulge initiation (b) and development (c), to differentiation of floral primordia (d). M: Meristem; YL: Young Leaf; IM: Inflorescence Meristem.

matched to a given protein exclusively was designated as an mSinglet (additional file 7). After such manual processing, the number of mUnigene (mSinglet + mContig) was lower than that generated from the original assembly (Table 1).

Cutting by $E$ value $<1 e-5$, more than $77 \%$ ESTs on average from each library matched to at least one entry in BlastX searching against the nonredundant protein database at NCBI. Based on the best match, 7802 records were retrieved, some of which were shared by two or more libraries. After removing those repetitive records across libraries, annotated mUnigenes were up to 6375 (Table 1). The number of records unique to each library was as follows: 1305 to the $0 \mathrm{~d}$ library, 1275 to the $(5+10) \mathrm{d}$ library, 1479 to the $(20+30) d$ library, and 1231 to the $40 \mathrm{~d}$ library. The absence of these sequences from other libraries is probably a result of inadequate reading of the sequences from each library. Overlapping analysis between libraries revealed that $4378 \mathrm{mUni}-$ genes were absent in the $0 \mathrm{~d}$ library, out of which 28 were shared by the three vernalized libraries (additional file 4). Proteins involved protein synthesis and postprocessing, including several ribosomal proteins, an RNA-binding protein, a subunit of transport SEC1 protein, and an ubiquitin fusion protein, were predominant in this shared gene set.

\subsection{Functional Annotation and Classification of D. nobile Uni-} genes. BLASTX searching showed that 78\%, 72\%, 70\%, and $42 \%$ aUnigenes from the four libraries matched at least one record in the TAIR10 protein database, respectively, ( $E$ value $<1 e-7)$. By manually removing the redundant records, the number of annotated unigenes, namely, mUnigenes, came to 
TABLE 1: Summary of the four libraries.

\begin{tabular}{|c|c|c|c|c|c|}
\hline Library name & $0 \mathrm{~d}$ & $(5+10) d$ & $(20+30) d$ & $40 \mathrm{~d}$ & Sum** \\
\hline Total EST & 3391 & 3369 & 4112 & 4145 & 15017 \\
\hline$a^{*}$ Singlet & $2704(79.74 \%)$ & $2736(81.21 \%)$ & $3166(77 \%)$ & $3310(79.86 \%)$ & \\
\hline aContig & 272 & 247 & 325 & 338 & \\
\hline aUnigene & $2976(87.76 \%)$ & $2983(88.54 \%)$ & $3491(84.9 \%)$ & $3648(88 \%)$ & \\
\hline Mean length of EST (bp) & 654 & 615 & 588 & 745 & \\
\hline \multicolumn{6}{|c|}{ BlastX against nonredundance protein database $(\mathrm{Nr})$ at NCBI } \\
\hline No hit aUnigene & 429 & 565 & 770 & 1477 & 3241 \\
\hline Hit aUnigene & 2547 & 2418 & 2721 & 2171 & \\
\hline $\mathrm{m}^{*}$ Unigene $^{\S}$ & 2426 & 2520 & 2985 & 3112 & \\
\hline mSinglet & 2016 & 2145 & 2539 & 2657 & \\
\hline mContig & 410 & 375 & 446 & 455 & \\
\hline mUnigene ${ }^{\S \S}$ (Nonredundant a & ss libraries) & 6375 & & & \\
\hline mSinglet & & $5290(82.98 \%)$ & & & \\
\hline mContig & & $1085(17.02 \%)$ & & & \\
\hline Total mUnigene & & 9616 (sum of 6375 and 3241) & & & \\
\hline \multicolumn{6}{|c|}{ Blast $X$ against TAIR9 protein datasets at TAIR } \\
\hline No hit aUnigene & 651 & 812 & 1032 & 2087 & \\
\hline Hit aUnigene & $2325(78 \%)$ & $2171(72 \%)$ & $2459(70 \%)$ & $1561(42 \%)$ & \\
\hline mUnigene ${ }^{\S}$ & 1599 & 1553 & 1756 & 1163 & \\
\hline GO annotated mUnigene ${ }^{\S}$ & 1433 & 1403 & 1596 & 997 & \\
\hline
\end{tabular}

* "a" is the first letter of "assembly," indicating the sequence is generated from assembly using Phrap.

" $m$ " is that of "manual," indicating the sequence is generated from manually removing the redundancies.

** This column lists simple sum of the 4 libraries.

$\S$ and $\S \S$ The nonredundant Unigene in the row marked “§” are nonredundant within a library but maybe redundant across libraries, while those marked

“§§” are nonredundant across libraries.

$1599,1553,1756$, and 1163 for the four libraries, respectively. GO terms were retrieved for approximately $89 \%$ of these Unigenes (Table 1). To develop a clear, functional classification for the annotated mUnigenes, the initial GO terms were first converted to GOSlim-plant terms and the frequency of each GOSlim-plant term was calculated as described in Section 2. The overall pattern of distribution of Unigenes in D. nobile was similar to that in Arabidopsis (Figure 2). However, the categories "response to stress," "response to biotic and abiotic stimulus," and "electron transport and energy pathway" were more pronounced in D. nobile. Low-temperature treatment seems to lead to small changes in D. nobile. Vernalization-induced increase in the frequency of categories "DNA and RNA metabolism" and "signal transduction" was less than 2 folds, whereas no obvious changes were observed in the frequency of "cell organization and biogenesis," "development process," "electron transport or energy pathways," and "protein metabolism." These results implied that $D$. nobile would maintain a constant transcription level during vernalization. Similar observations were previously reported in wheat, in which the transcript accumulation in crown tissue was lower in winter varieties than that in spring varieties during cold acclimation [30].

3.3. Functional Enrichment in Vernalized D. nobile. To identify the overrepresented gene categories, we performed a functional enrichment analysis using BinGO software [27].
Referring to the whole annotation of Arabidopsis transcriptome (TAIR10), the gene category "response to stress" was overrepresented in the axillary buds, considering either before or after exposure to low temperature. This phenomena was likely linked to cold, high salinity, and heat stresses (data no shown), and the cold-induced responses may be the major theme because cold-responsive genes were increased dramatically in the total number with time during the whole vernalization process. Among the cold-responsive gene set, a homolog of Arabidopsis AGL19, a key regulator in vernalization-associated flowering, was presented, and transcript quantification confirmed that the initial induction of this AGL19 homolog occurred just after 5 days of vernalization (Figure 5), implying that transition to the flowering phase would be initiated early by vernalization. Forty days of vernalization is probably in relation to cold adaptation, as two cold-acclimation-related genes, one similar to Arabidopsis CAX1 and the other to ATRZ-1A [31], were induced at this stage. These observations suggested a link between the initiation of floral transition and the cold acclimation in D. nobile. Besides, responses to oxidative stress, zinc ion, and light stimulus were also remarkable, but they were vernalization-stagespecific and could not be retained when low-temperature exposure was extended over a longer duration (Table 2). Genes involved in metabolism of carbon, nucleotide, and amino acid were also enriched before vernalization and in the early stage of low-temperature treatment (Table 2). 
TAвLE 2: Significantly over-/under-represented GO identifiers specifically to a stage of vernalizaiton (corrected $P$ value $<1 e-05$ ).

\begin{tabular}{llc}
\hline & Overrepresented & Under-represented \\
\hline $0 \mathrm{~d}$ & Response to stimulus & Gene expression \\
& Response to zinc ion & Translational elongation \\
Metabolism process & Gene expression \\
& Purine ribonucleotide metabolic process & Translational elongation \\
& Methionine metabolic process & Gene expression \\
$(5+10) \mathrm{d}$ & Metabolism process & Translational elongation \\
& Carbon utilization & Metabolic compound salvage \\
$(20+30) \mathrm{d}$ & Localization & None \\
& Protein transport & Response to stimulus \\
& Denfense response to bacterium & Response to light stimulus \\
& Gene expression & Translation \\
& Cellular component organization and biogenesis & Ribosome biogenesis \\
Nucleosome assembly &
\end{tabular}

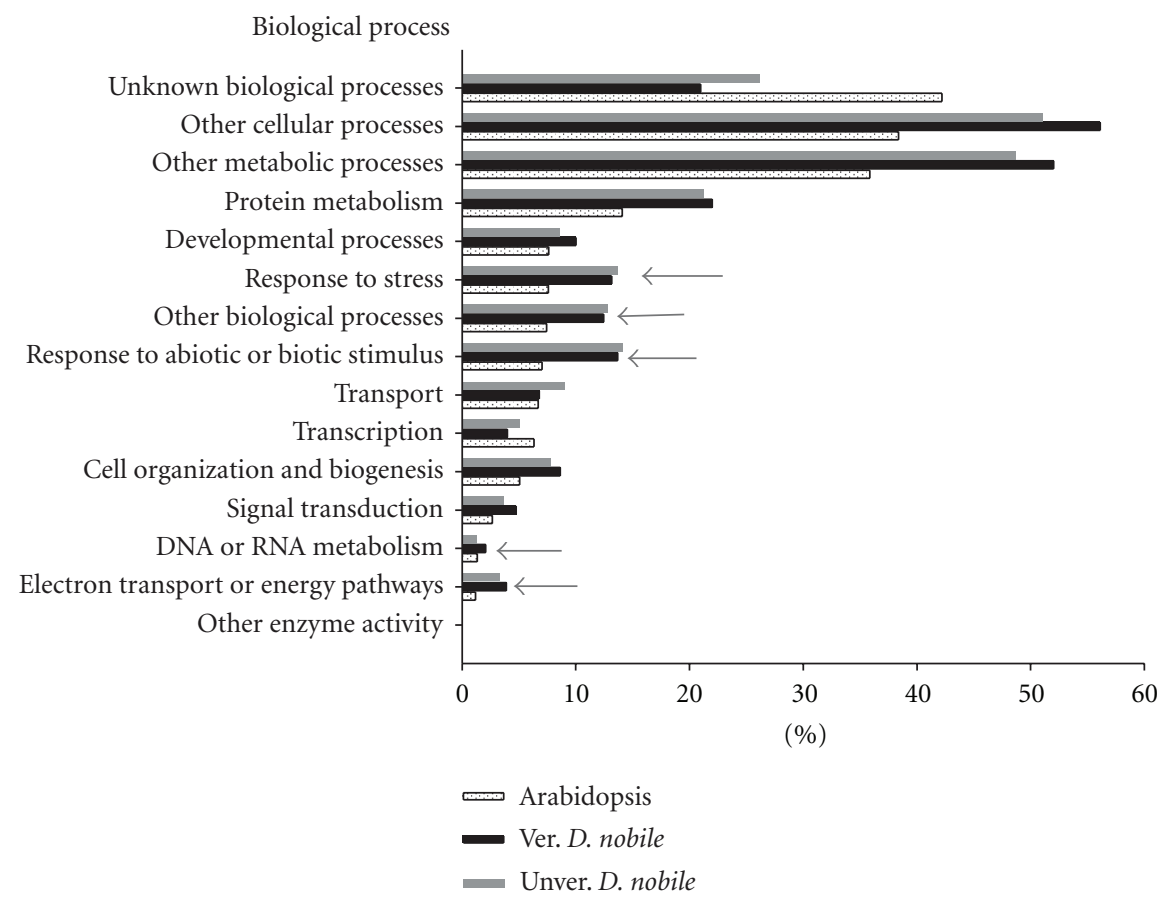

FIGURE 2: Classifications of unigenes based on GOSlim-plant ontology of biological process. GO terms were assigned to D. nobile unigenes according to best hit in BLASTX against Arabidopsis protein database (http://www.arabidopsis.org/). Classification was conducted at http://www.arabidopsis.org/tools/bulk/go/index.jsp for unvernalized (grey bars) and vernalized (black bars) D. nobile gene sets according to GOSlim-plant annotations on biological process. Spectrums of unigene categories were similar between these two gene sets. Unigenes that grouped into the "unknown biological process" category are $26.17 \%$ and $21.01 \%$ of the vernalized and unvernalized dataset, respectively. The Arabidopsis whole annotation is used as a reference (dotted bars). Gene categories with approximate 2-fold increase in D. nobile relative to that in Arabidopsis are indicated by arrows. 


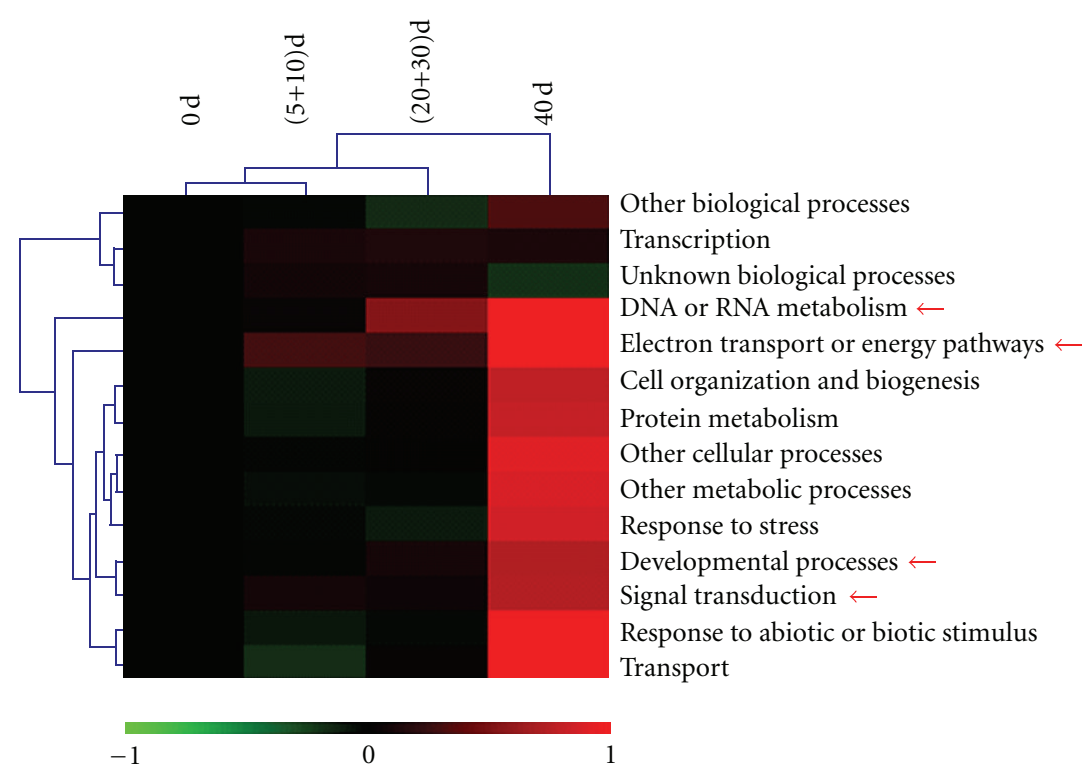

FIGURE 3: Time course profile of gene categories (GOSlim-plant terms). Hierarchical clustering analysis was performed to monitor the timecourse profiles of gene categories basing on the fold changes relative to $0 \mathrm{~d}$. The arrows indicate the gene categories that were initially induced after short term vernalization and activated after long-term vernalization.

Differing from the changes on stress-related responses and metabolism status, regulations of translation, protein synthesis, and chromatin assembly became activated after a long-term of vernalization, indicating posttranscriptional regulation might have important roles in low-temperatureinduced responses in D. nobile.

The "flower development" subcategory was not overrepresented as expected, which led to a speculation that transition to the flowering stage would not occur, but we found that the frequency of this subcategory increased gradually with time during the vernalization process, and many homologs involved in various pathways promoting the floral transition and flowering development were induced after the low-temperature treatment. This suggested that the transition may have begun although it was not detected at this stage, which is reasonable because exposure to low temperature for 30-35 days is enough to promote flowering in D. nobile.

3.4. Time-Course Change in Biological Processes in D. nobile during Vernalization. The increasing number of overrepresented categories implies that the overall transcription is activated as vernalization proceeds (Table 2). Clustering analysis revealed that some GOSlim-plant categories had followed such a trend, including "DNA and RNA metabolism," "development processes," "electron transport and energy pathways," and "signal transduction" (Figure 3). Besides, the $0 \mathrm{~d}$ set was initially grouped with the $(5+10)$ dataset, indicating that the transcript profile at the initial stage of vernalization was similar to that during vegetative development. Midway through vernalization (the $(20+30)$ dataset), genes involved in "DNA and RNA metabolism," "development process," and "electron transport and energy pathways" were induced (Figure 3 ). The final stage (40 days of vernalization) was clearly distinct and showed greater and more extensive induction of genes (Figure 3). Together, these results suggest two types of transcriptional regulation during vernalization in D. nobile, cellular homeostasis at the early stage and systematic activation at the late stage.

3.5. Homologs of Cereal Vernalization-Responsive Genes. Based on the functional prediction and enrichment analysis, we identified 59 D. nobile genes whose Arabidopsis homologs are annotated involving the flowering development or are related to development of floral organs (Table 3 shows 13 genes as examples). We initially focused on those putative orthologs and functional equivalents that have been reported to be involved in vernalization or those relevant pathways.

Cereal VRN1, together with VRN2, VRT2, and VRN3, forms the major regulation pathway that controls vernalization-induced flowering in temperate cereals $[1,2]$, in which VRN1 and VRN3 serve as flowering activators while VRN2 and VRT2 as repressors. TaVRT2 represses transcription of the wheat vernalization gene TaVRN1. Cereal VRN2 is similar to the Arabidopsis $\mathrm{CO}$ and CO-like proteins, containing a CONSTANS, CONSTANS-like, and TOC (CCT) domain [32]. It acts as a negative regulator in flowering and is suppressed after vernalization. Heterologous expression of TaVRN2 can delay flowering in transgenic Arabidopsis [33]. However, no orthologs of the TaVRN2 locus have been found in Brassicaceae [15], and the origin and evolution of VRN2 in wheat and other monocots remain a mystery. In the present study, we did not identify the VRN2 ortholog in D. nobile, possibly duo to limits of techniques.

However, several EST sequences similar to VRN2's interactor, VRT2, were identified. Cereal VRT2 is homologous to 
TABLE 3: Thirteen putative genes of $D$. nobile involved in floral induction.

\begin{tabular}{|c|c|c|c|c|c|}
\hline \multirow{2}{*}{ Putative gene } & \multirow{2}{*}{ Arabidopsis homologue } & \multicolumn{4}{|c|}{ EST number } \\
\hline & & $0 \mathrm{~d}$ & $(5+10) \mathrm{d}$ & $(20+30) d$ & $40 \mathrm{~d}$ \\
\hline DnVRN1 & AP1, cereal VRN1 & 0 & 4 & 0 & 0 \\
\hline DnTSF & $F T, T S F$ & 0 & 1 & 0 & 0 \\
\hline$D n F T$ & FT, TSF & 0 & 0 & 0 & 1 \\
\hline CO-like & CO-like 4 & 5 & 0 & 2 & 0 \\
\hline DnAGL19 & AGL19, SOC1 & 0 & 2 & 1 & 0 \\
\hline DnEMF2 & $E M F 2$ & 0 & 0 & 0 & 1 \\
\hline DnMSI1 & MSI1 & 1 & 0 & 0 & 1 \\
\hline$D n C L F$ & $C L F$ & 0 & 0 & 0 & 2 \\
\hline$F C A$ & $F C A$ & 1 & 0 & 0 & 0 \\
\hline FVE & FVE & 1 & 1 & 1 & 0 \\
\hline$F L K$ & $F L K$ & 0 & 1 & 0 & 0 \\
\hline VRT2-like & SVP, AGL24, HvVRT2 & 2 & 2 & 1 & 0 \\
\hline VIP-like & VIP4 & 0 & 1 & 0 & 0 \\
\hline
\end{tabular}

Arabidopsis SVP and AGL24 and possibly downregulated by vernalization $[34,35]$. Transcription of the D. nobile VRT2like homolog was not repressed but activated by low temperature (Figure 5), which is different from what is observed in wheat VRT2 but mimics the behaviour of AGL24 in Arabidopsis [36]. The role of this D. nobile VRT2-like gene is not clear, and further studies are necessary to ascertain its involvment in vernalization-induced flowering.

Vernalization-associated repression of VRN2 transcription in some cereals is probably linked to the induction of VRN1, a flowering activator [37]. Cereal VRN1 is the ortholog of Arabidopsis AP1, an A-class gene that functions in identifying floral meristem [38]. In temperate cereals, it has more functions such as cold signal transmission during vernalization [2]. The gene is initially transcribed at low levels and induced by vernalization under both long- and shortday conditions $[39,40]$. Activation of this gene can subsequently induce FT/VRN3 expression in leaves, which makes plants be capable of flowering (Figure 6). Therefore, VRN1 has been suggested to be the primary regulator of vernalization-mediated floral transition in temperate cereals. Orthologs of VRN1 have been isolated from many monocots, including wheat, barley, oat, and rye [40-42]. A D. nobile homolog of VRN1 was also identified in this study. This gene codes for a protein consisting of 247 amino acids and has a typical MADS-box domain located at the $5^{\prime}$-end (Figure 4). Pair wise comparisons indicated that the deduced peptide sequence had $48.5 \%, 53.8 \%$, and $61.5 \%$ overall identities with AtAP1 (AGL7), AtFUL (AGL8), and TmVRN1 (AAZ76882.1) (additional file 6), respectively, and we designated it, DnVRN1, to indicate its close relation to cereal VRN1. Transcription of this gene in buds was initially induced after vernalization for 5 days, remained constant thereafter, and decreased slightly after long-term vernalization (Figure 5 ). This pattern of expression was somewhat similar to that of VRN1 in wheat and barley [42-44].
In addition to VRN1, cold-induced reduction of VRN2 is also linked, at least in barley, to PPD1-dependent induction of VRN3, an ortholog of Arabidopsis FT [11, 16]. FT/VRN3 may be expressed in leaves and the protein is then translocated to the shoot apex, where it triggers the transition to flowering [45]. Two VRN3-like transcripts were identified from the axillary buds of $D$. nobile. The first one codes for a protein of 178 amino acids similar to Arabidopsis FT and TSF with identities of $57.3 \%$ and $56.7 \%$, respectively, (Figure 4 and additional file 6). When compared with OsFT (rice Hd3a, BAB61030), OsTSF (NP_001046372), and Triticum aestivum VRN3 (TABK32208.1), the amino acid identity rose to $59.2 \%, 62.4 \%$, and $55.6 \%$, respectively. Phylogenetic analysis also indicated it was closer to OsTSF than to OsFT (additional file 6). Thus, we named this gene DnTSF. Only one EST of this gene was observed in the $(5+10) d$ library (Table 3$)$, but real-time qPCR assay showed that it was induced in buds throughout the entire duration of vernalization (Figure 5). Another VRN3-like transcript was identified from the $40 \mathrm{~d}$ library (Table 3 ). The full-length cDNA of this transcript was also indentified (Figure 4). Phylogenetic analysis revealed that it was close to OsFT, so we designate it DnFT (additional file 6). The expression level of DnFT in buds remained at very low level under vernalization (Figure 5), unlike that of DnTSF. In leaves, however, the expression of DnFT was lower before vernalization (Liang, S, unpublished data) and was induced to a higher level by vernalization $(\mathrm{Li}, \mathrm{R}-\mathrm{H}$, unpublished data). Together, these results led to a speculation that vernalization would induce DnFT in leaves, and the protein then be translocated to the shoot meristem to serve as florigen. Thus, the expression and the function of DnFT may be similar to their orthologs in Arabidopsis, wheat, and barley [46]. DnTSF, on the other hand, is probably regulated by means distinct from those in relation to DnFT and may form a part of another pathway as that being proposed in Arabidopsis [47]. 


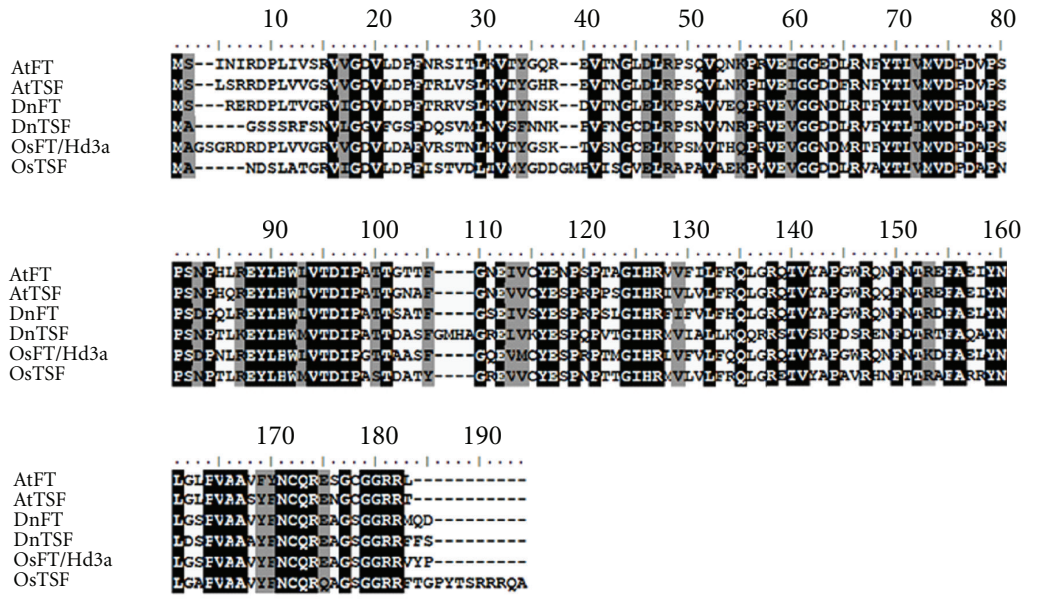

(a)

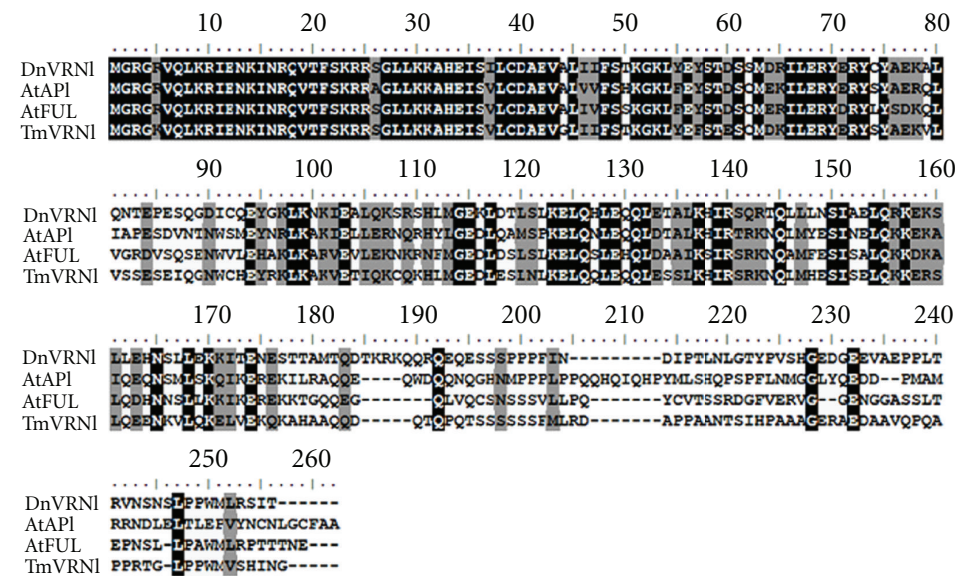

(b)

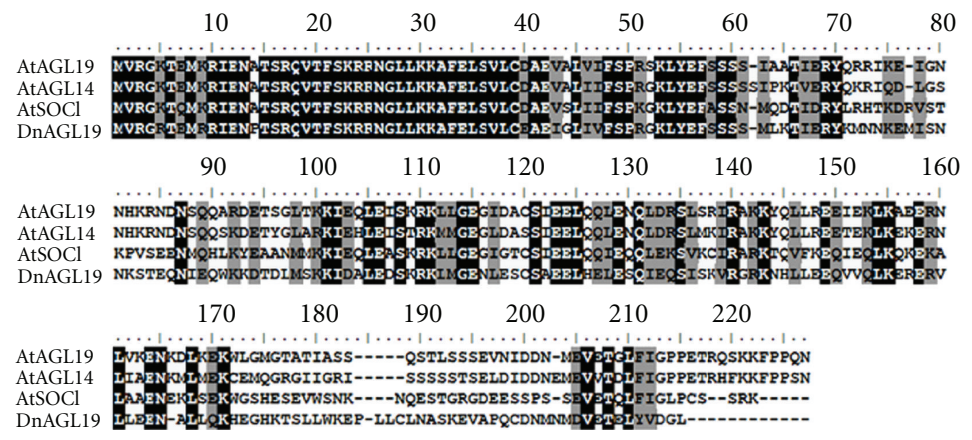

(c)

FIGURE 4: Alignment of FT, VRN1, and AGL19 homologous proteins. Phylogentic analyses are shown in additional file 6. (a) DnFT and DnTSF are aligned with FT homologs from Arabidopsis (At) and rice (Os). Phylogenetic analysis indicates that DnTSF is closer to OsTSF while DnFT is to OsFT. (b) DnVRN1 is compared with Arabidopsis AP1, FUL and wheat VRN1. (c) DnAGL19 is aligned to Arabidopsis AGL19, AGL14, and SOC1. Distance (branch length) between DnAGL19 and AtAGL19 is less than that between it and AtSOC1. Identical sites are shaded in black, while those similar sites are in grey.

To summarize, we identified $D$. nobile homologs of cereal vernalization-responsive genes, including two essential components, DnVRN1 and DnFT, and the VRT2-like gene (Figure 6). Vernalization induced the transcription of DnVRN1 in axillary buds where the inflorescence would emerge later (Figure 5), suggesting that DnVRN1, like its homologous gene in cereal [42], would involve in the transition to flowering. Another component, DnFT protein, was probably synthesized in leaves and translocated to the shoot meristem after vernalization where the feedback 


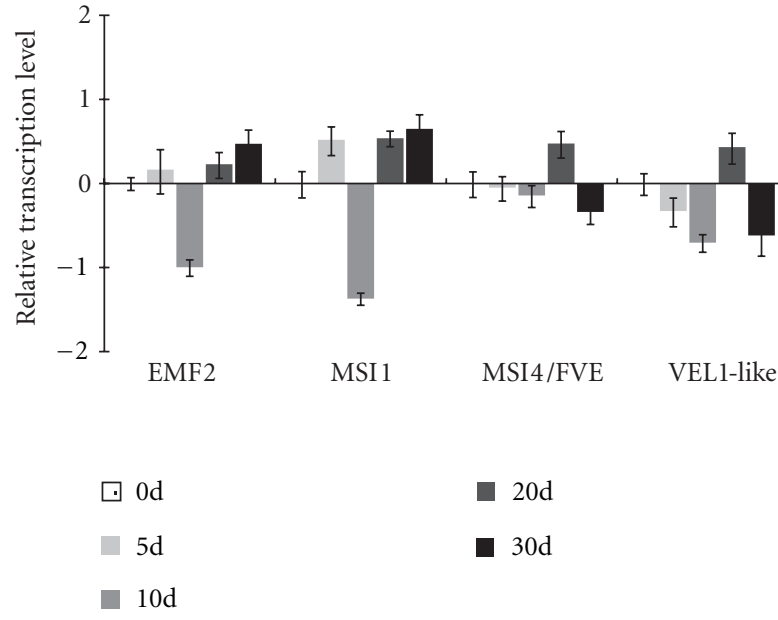

(a)

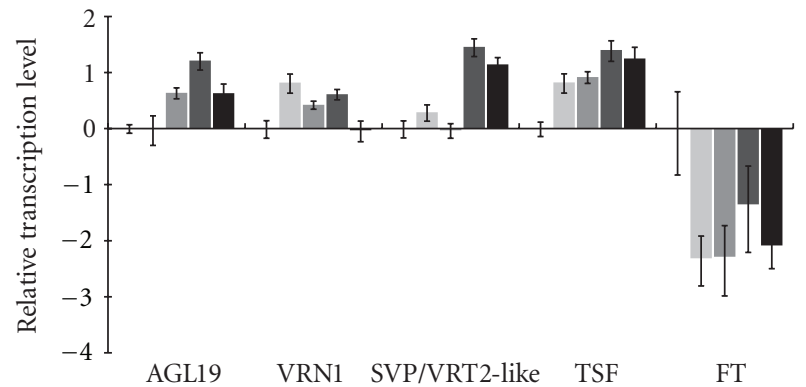

$$
\begin{aligned}
& \square 0 \mathrm{~d} \\
& -5 \mathrm{~d} \\
& \square \quad 10 \mathrm{~d}
\end{aligned}
$$

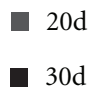

$20 \mathrm{~d}$

$30 \mathrm{~d}$

Figure 5: Time-course transcript profiles of selected flowering-relevant genes during the vernalization process. Relative expression levels of each selected genes after exposing at $15^{\circ} \mathrm{C} / 10^{\circ} \mathrm{C}$ (day/night) for $0,5,10,20$, and 30 days are shown. Total RNA were isolated and were reverse transcribed to cDNA that served as templates in real-time qPCR assays. RQ values of each gene relative to $0 \mathrm{~d}$-bud samples were $\log 2$-transformed ( $y$-axis) and plotted against the time periods ( $x$-axis). The positive and negative values indicate the vernalization-induced activation and repression, respectively. The error bars are calculated based on the (RQMin and RQMax) confidence interval (95\%).

loop between DnVRN1 and DnFT is established. The close homolog of DnFT, namely, DnTSF, probably regulated flowering in $D$. nobile in a way distinct from that followed by DnFT. Our observations pointed to a VRN1-mediated pathway in $D$. nobile which evolutionarily conserved between this species and temperate cereals. However, this possibility needs to be confirmed by further studies.

3.6. Absence or Repression of FLC-Dependent Pathway in Vernalized D. nobile. FLC is considered as the central component in vernalization-induced floral transition in Arabidopsis [2]. However, the D. nobile FLC homolog was not found in our dataset, even in the $0 \mathrm{~d}$ samples being expected to contain high levels of FLC transcripts. One possible explanation for the absence of FLC transcript is that the orthologous gene of Arabidopsis FLC did not exist in D. nobile genome per se, like cases in some temperate cereals [37].

However, an alternative explanation that D. noble FLC homolog was repressed by vernalization is also reasonable. Some transcripts involved in FLC-dependent vernalization responses were found in vernalized $D$. nobile (Table 3 ), including two positive regulators of FLC, homologs of VIP4 and VIP5 [48, 49]; an interaction partner of FLC, SVP [50]; and some components relevant to FLC chromatin histone modification, such as CLF, MSI1, UBC1, UBC2, HTA9, and HUB1 [2]. These observations, together with the absence of FLC transcript, implied that an FLC homolog would exist in $D$. nobile but would be in fact repressed by activation of autonomous pathway in axillary buds (indicated by the higher level of transcription of FVE after exposure to lowtemperature, Table 3 and Figure 5) or by increasing enrichments of H3K27 methylation on the FLC locus through activities of polycomb repressive complex 2 (PRC2, Table 3) [1, 3]. This repression consequently led to lower level of FLC expression and made it difficult to identify its transcript through the experimental techniques used in present study.

3.7. Autonomous Pathway Homologs. An autonomous pathway represses the transcription of FLC in Arabidopsis [51]. Components of this pathway, including FCA, FVE (aka MSI4), and FLK, were also identified from $D$. nobile buds. $D$. nobile FVE homolog was probably expressed constitutively in buds of $D$. nobile (Figure 5), indicating that it was not affected by vernalization. The level of $D$. nobile FCA transcript may decrease during the course of vernalization (Table 3), but the effect of this decrease could not be assessed in the present study and should be confirmed by further experiments.

3.8. Homologs Involved in Chromatin Histone Methylation. Polycomb repressive complex 2 contributes to the maintenance of chromatin $\mathrm{H} 3 \mathrm{~K} 27$ methylation in plants. The VRN2-containing PRC2 complex (not the equivalent of cereal VRN2) consistently suppresses the expression of FLC in Arabidopsis. However, VRN2 homologs were not found in rice [8], nor could we find in the D. nobile EST collection, which suggested that this gene, as well as the VRN2-containing PRC2 complex, may be absent in this Orchidaceae species. On the other hand, the EMF2-containg PRC2 complex probably play a role in the response to vernalization in D. nobile. The D. nobile EMF2 homolog and two other genes, CLF and MSI1, were transcribed in buds (Figure 4), and vernalization-induced transcription of these proteins may be related to $\mathrm{H} 3 \mathrm{~K} 27$ methylation of some gene loci, possibly, including the AGL19 locus, but not to that of FLC [5].

3.9. AGL19 Homolog and AGL19-Mediated FLC-Independent Pathway. Two FLC-independent pathways, one mediated by 


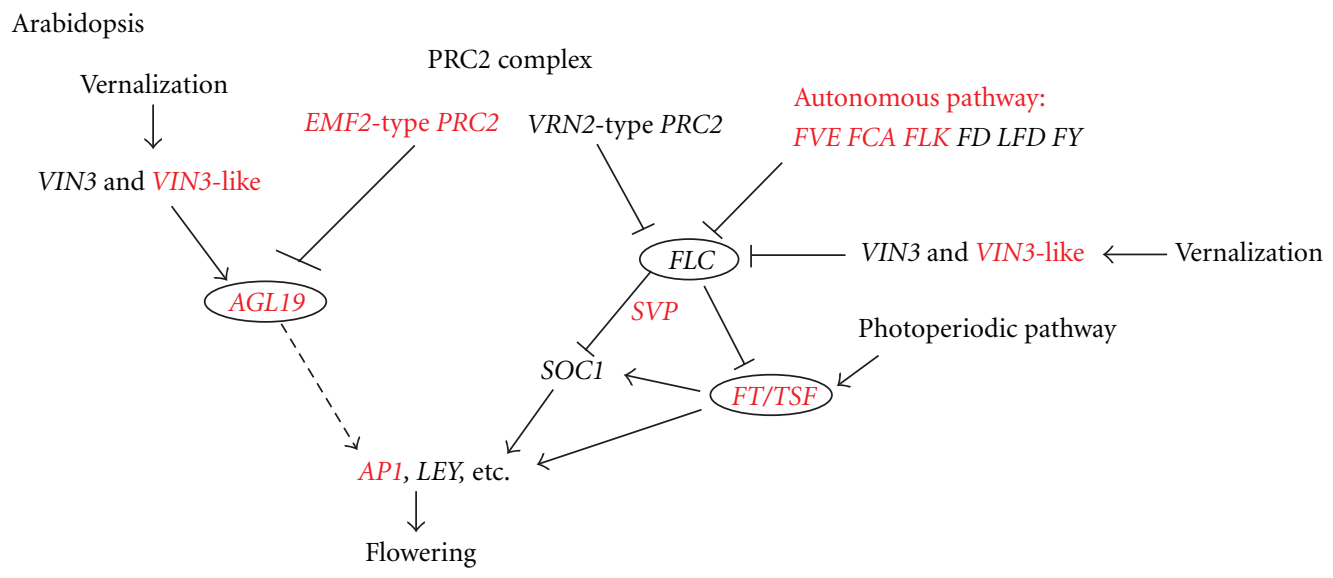

Cereal PRC2 complex: histone methylation

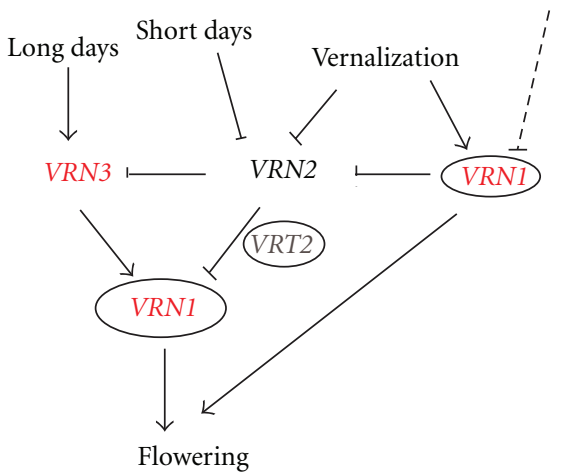

D. nobile

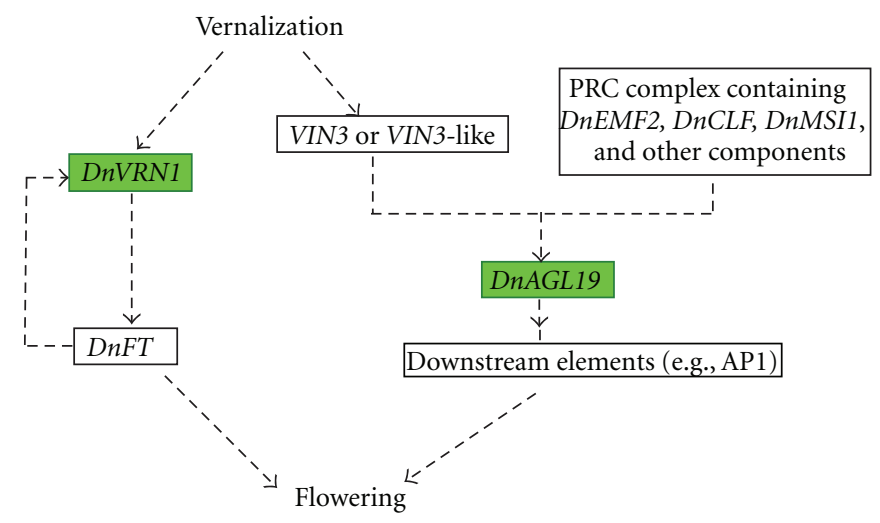

Figure 6: Predicted vernalization regulation networks in D. nobile. By comparative analysis with Arabidopsis and temperate cereal, we found many $D$. nobile unigenes induced by vernalization are homologs or functional equivalents of the flowering regulators. These homologs (highlighted in red) involve two vernalization pathways, one is the Arabidopsis AGL19 pathway and the other is the cereal VRN1 pathway. FLC homolog cannot be identified from $D$. nobile and this indicates that FLC mediated regulation might be absent or be repressed during vernalization process. Networks to regulate flowering by the vernalization, similar to those in Arabidopsis and temperate cereal, are proposed in D. nobile. It should be noted that this network must be confirmed by more extensive studies basing on more solid evidences. The network chats of Arabidopsis and temperate cereal are adopted from the article by Alexandre and Hennig [1], or are summarized according to the descriptions in articles by Higgins et al. [8], Kim et al. [2], and Trevaskis et al. [9, 10].

AGL19 and the other by AGL24, can promote the transition to flowering in Arabidopsis [1,2]. The absence of FLC transcripts in D. nobile implied that FLC-independent vernalization pathway(s) would play important roles in response to vernalization. It is noteworthy that a vernalization-induced transcript (Table 3, Figure 5) had 52.7\% and 48.8\% similarities at the level of amino acids to Arabidopsis AGL19 and SOC1, respectively, (Figure 4, additional file 6). Despite such close similarities, the deduced peptide of this transcript diverged more from AtSOC1 than AtAGL19, suggesting that 
the peptide is closer to AGL19. We, therefore, designated it DnAGL19. Transcription of DnAGL19 was activated after 20 days of vernalization (Figure 5), a situation somewhat similar to that of its homolog in Arabidopsis [5]. On the other hand, transcription of DnAGL19 was probably related to the EMF2-containg PRC2 complex (Figure 6). Expression of the D. nobile EMF2 and MSI1 homolog was suppressed early on during vernalization (10 days) and increased slightly thereafter (Figure 5). Repression of these genes early on would be related to induction of DnAGL19 (Figure 5) and implied that the EMF2-complex was involved in the regulation of DnAGL19 by histone methylation. A long period of vernalization may help in restoring the transcription levels of EMF2 and MSI1, which may in turn lead to the re-establishment of $\mathrm{H} 3 \mathrm{~K} 27 \mathrm{~m} 3$ at some gene loci. In brief, a DnAGL19-involved regulation network, similar to the Arabidopsis AGL19 pathway, may be conserved in the monocotic D. nobile. However, more experimental studies are needed to confirm this possibility.

\section{Conclusions}

From a collection of ESTs derived from buds of vernalized D. nobile plants, 9616 nonredundant unigene sequences were identified, of which approximately $64 \%$ matched at least one record in the nonredundant protein database at NCBI or in the Arabidopsis TAIR10 protein dataset. GO annotations based on ontology of biological process were assigned to each unigene according to the best match in BLASTX. Three libraries, corresponding to critical lengths (number of days, $\mathrm{d}$ ) of vernalization $((5+10) \mathrm{d},(20+30) \mathrm{d}$, and $40 \mathrm{~d})$, provided insights into changes in gene categories. As expected, genes including those in the "response to cold" category were significantly predominant during vernalization, and genes that control flowering time were initially induced early on. These results indicate that vernalization causes dynamic changes in the transcriptome and induces flowering in D. nobile, which may crosstalk with cold acclimation.

The absence of FLC in the EST collection indicated that, as in other monocots, an FLC-dependent pathway may not exist in D. nobile. However, this requires confirmation by extensive evidences. Close homologs of vernalization-responsive genes of temperate cereals, including the DnVRN1 and DnFT, as well as a homolog similar to Arabidopsis AGL19, were identified, pointing to the presence of similar regulation networks in vernalization-induced transition to the flowering in D. nobile (Figure 6).

Information on preliminary networks regulating the transition in D. nobile (Figure 6) will facilitate further characterization of the key players in vernalization pathways in Orchidaceae. Our findings pave the way to greater understanding of the vernalization network in D. nobile, which will provide novel insights not only on the mechanism that controls flowering time but also on the genetic basis underlying the evolutionary adaptation to cold in Orchidaceae. Finally, exploring the genes that control flowering time will be hoped to benefit in molecular breeding of $D$. nobile for commercially successful cultivars in future.

\section{Abbreviations}

$\begin{array}{ll}\text { D. nobile: } & \text { Dendrobium nobile } \\ \text { VRN1: } & \text { VERNALIZATION 1 } \\ \text { AGL9: } & \text { AGAMOUS-LIKE 19 } \\ \text { FLC: } & \text { Flowering Locus C } \\ \text { VIN3: } & \text { VERNALIZATION INSENSITIVE } 3 \\ \text { VEL: } & \text { VERNALIZATION 5/VIN3-LIKE } \\ \text { PRC2: } & \text { Polycomb Repression Complex } 2 \\ \text { FT: } & \text { Flowering Locus T } \\ \text { SOC1: } & \text { Suppressor of Overexpression of CO 1 } \\ \text { H3K27m3: } & \text { Histone H3 Lys 27 (H3K27) } \\ & \text { trimethylation } \\ \text { CLF: } & \text { Curly Leaf } \\ \text { MSI1: } & \text { Multicopy Supressor of IRA 1 } \\ \text { MAF2: } & \text { MADS AFFECTING FLOWERING } 2 \\ \text { VIP4: } & \text { Vernalization Independence } 4 \\ \text { SEC1: } & \text { Similar to Electron Career 1 } \\ \text { CAX1: } & \text { Cation Exchanger 1 } \\ \text { CO: } & \text { Constans } \\ \text { COL: } & \text { Constans Like } \\ \text { FUL: } & \text { FRUITFUL } \\ \text { AP1: } & \text { Apetala 1 } \\ \text { TSF: } & \text { Twin Sister of FT } \\ \text { SVP: } & \text { Short Vegetative Phase } \\ \text { UBC: } & \text { Ubiquitin Conjugating enzyme } \\ \text { HUB: } & \text { Histone Monoubiquitination } \\ \text { FLK: } & \text { Flowering Locus KH Domain } \\ \text { EMF2: } & \text { Embryonic Flower 2. }\end{array}$

\section{Acknowledgments}

The authors sincerely thank Chengwei Yang, Qili Feng, and Haihang Li for their helpful comments and suggestions to improve this study and the manuscript. They thank Yang Shen and Xiao-Shu Chen (Sun Yat-San University, China) for their assistance in EST analysis. We thank Wuxia Guo, Minyan Chen, Youqiao Chen, Jie Xu, Jin Liu, Hehu Liu, Lei Chen, and Zhuanying Yang for their help in sample collection. This study is supported by National Natural Science Foundation of China (Grant no. u0731006, 31171994) and Natural Science Foundation of Guangdong Province (10451063101005250, 2011A020201008, 2011A020102007).

\section{References}

[1] C. M. Alexandre and L. Hennig, "FLC or not FLC: the other side of vernalization," Journal of Experimental Botany, vol. 59, no. 6, pp. 1127-1135, 2008.

[2] D. H. Kim, M. R. Doyle, S. Sung, and R. M. Amasino, "Vernalization: winter and the timing of flowering in plants," Annual Review of Cell and Developmental Biology, vol. 25, pp. 277-299, 2009.

[3] C. C. Wood, M. Robertson, G. Tanner, W. J. Peacock, E. S. Dennis, and C. A. Helliwell, "The Arabidopsis thaliana vernalization response requires a polycomb-like protein complex that also includes VERNALIZATION INSENSITIVE 3," Proceedings of the National Academy of Sciences of the United States of America, vol. 103, no. 39, pp. 14631-14636, 2006. 
[4] S. D. Michaels and R. M. Amasino, "Flowering Locus C encodes a novel MADS domain protein that acts as a repressor of flowering," Plant Cell, vol. 11, no. 5, pp. 949-956, 1999.

[5] N. Schönrock, R. Bouveret, O. Leroy et al., "Polycomb-group proteins repress the floral activator AGL19 in the FLC-independent vernalization pathway," Genes and Development, vol. 20, no. 12, pp. 1667-1678, 2006.

[6] O. J. Ratcliffe, R. W. Kumimoto, B. J. Wong, and J. L. Riechmann, "Analysis of the Arabidopsis MADS AFFECTING FLOWERING gene family: MAF2 prevents vernalization by short periods of cold," Plant Cell, vol. 15, no. 5, pp. 1159-1169, 2003.

[7] H. Yu, Y. Xu, E. L. Tan, and P. P. Kumar, "AGAMOUS-LIKE 24, a dosage-dependent mediator of the flowering signals," Proceedings of the National Academy of Sciences of the United States of America, vol. 99, no. 25, pp. 16336-16341, 2002.

[8] J. A. Higgins, P. C. Bailey, and D. A. Laurie, "Comparative genomics of flowering time pathways using Brachypodium distachyon as a model for the temperate Grasses," PLOS ONE, vol. 5, no. 4, Article ID e10065, 2010.

[9] B. Trevaskis, M. N. Hemming, E. S. Dennis, and W. J. Peacock, "The molecular basis of vernalization-induced flowering in cereals," Trends in Plant Science, vol. 12, no. 8, pp. 352-357, 2007.

[10] B. Trevaskis, M. Tadege, M. N. Hemming, W. J. Peacock, E. S. Dennis, and C. Sheldon, "Short Vegetative Phase-like MADSBOX genes inhibit floral meristem identity in barley," Plant Physiology, vol. 143, no. 1, pp. 225-235, 2007.

[11] L. Yan, D. Fu, C. Li et al., "The wheat and barley vernalization gene VRN3 is an orthologue of FT," Proceedings of the National Academy of Sciences of the United States of America, vol. 103, no. 51, pp. 19581-19586, 2006.

[12] C. Li and J. Dubcovsky, "Wheat FT protein regulates VRN1 transcription through interactions with FDL2," Plant Journal, vol. 55, no. 4, pp. 543-554, 2008.

[13] S. N. Oliver, E. J. Finnegan, E. S. Dennis, W. J. Peacock, and B. Trevaskis, "Vernalization-induced flowering in cereals is associated with changes in histone methylation at the VERNALIZATION 1 gene," Proceedings of the National Academy of Sciences of the United States of America, vol. 106, no. 20, pp. 83868391, 2009.

[14] B. Trevaskis, M. N. Hemming, W. J. Peacock, and E. S. Dennis, "HvVRN2 responds to daylength, whereas HvVRN1 is regulated by vernalization and developmental status," Plant Physiology, vol. 140, no. 4, pp. 1397-1405, 2006.

[15] L. Yan, A. Loukoianov, A. Blechl et al., "The Wheat VRN2 Gene Is a Flowering Repressor Down-Regulated by Vernalization," Science, vol. 303, no. 5664, pp. 1640-1644, 2004.

[16] M. N. Hemming, W. J. Peacock, E. S. Dennis, and B. Trevaskis, "Low-temperature and daylength cues are integrated to regulate Flowering Locus T in barley," Plant Physiology, vol. 147, no. 1, pp. 355-366, 2008.

[17] G. Stocker, P. S. Lavarack, and W. Harris, Dendrobium and Its Relatives, Timber Press, Portland, Ore, USA, 2000.

[18] G. B. Rotor, "Daylength and temperature in relation to growth and flowering of orchids," Cornell University Agricultural Experiment Station Bulletin, vol. 885, pp. 3-47, 1952.

[19] Z. H. Wang, L. Wang, and Q. S. Ye, "High frequency early flowering from in vitro seedlings of Dendrobium nobile," Scientia Horticulturae, vol. 122, no. 2, pp. 328-331, 2009.

[20] C. J. Goh and J. Arditti, "Orchidaceae," in Handbook of Flowering, A. H. Halevy, Ed., pp. 309-336, CRC Press, Boca Raton, Fla, USA, 1985.
[21] M. Skipper, L. B. Johansen, K. B. Pedersen, S. Frederiksen, and B. B. Johansen, "Cloning and transcription analysis of an AGAMOUS- and SEEDSTICK ortholog in the orchid Dendrobium thyrsiflorum (Reichb. f.)," Gene, vol. 366, no. 2, pp. 266274, 2006.

[22] Y. Xu, L. L. Teo, J. Zhou, P. P. Kumar, and H. Yu, "Floral organ identity genes in the orchid Dendrobium crumenatum," Plant Journal, vol. 46, no. 1, pp. 54-68, 2006.

[23] H. Yu, Shu Hua Yang, and Chong Jin Goh, " $D O H 1$, a class 1 knox gene, is required for maintenance of the basic plant architecture and floral transition in orchid," Plant Cell, vol. 12, no. 11, pp. 2143-2159, 2000.

[24] H. Yu, H. Y. Shu, and J. G. Chong, "Spatial and temporal expression of the orchid floral homeotic gene DOMADS1 is mediated by its upstream regulatory regions," Plant Molecular Biology, vol. 49, no. 2, pp. 225-237, 2002.

[25] B. Ewing, L. Hillier, M. C. Wendl, and P. Green, "Base-calling of automated sequencer traces using phred. I. Accuracy assessment," Genome Research, vol. 8, no. 3, pp. 175-185, 1998.

[26] S. Kumar, K. Tamura, and M. Nei, "MEGA3: integrated software for molecular evolutionary genetics analysis and sequence alignment," Briefings in Bioinformatics, vol. 5, no. 2, pp. 150-163, 2004.

[27] S. Maere, K. Heymans, and M. Kuiper, "BiNGO: a cytoscape plugin to assess overrepresentation of gene ontology categories in biological networks," Bioinformatics, vol. 21, no. 16, pp. 3448-3449, 2005.

[28] K. J. Livak and T. D. Schmittgen, "Analysis of relative gene expression data using real-time quantitative PCR and the $2^{-\Delta \Delta C T}$ method," Methods, vol. 25, no. 4, pp. 402-408, 2001.

[29] J. Zhuang, S. Huang, S. Pan, and Q. Ye, "Construction and identification of a cDNA expression library from Dendrobium nobile," Acta Horticulturae Sinica, vol. 33, no. 4, pp. 895-897, 2006 (Chinese).

[30] M. O. Winfield, C. Lu, I. D. Wilson, J. A. Coghill, and K. J. Edwards, "Plant responses to cold: transcriptome analysis of wheat," Plant Biotechnology Journal, vol. 8, no. 7, pp. 749-771, 2010.

[31] Y. O. Kim and H. Kang, "The role of a zinc finger-containing glycine-rich RNA-binding protein during the cold adaptation process in Arabidopsis thaliana," Plant and Cell Physiology, vol. 47, no. 6, pp. 793-798, 2006.

[32] S. Griffiths, R. P. Dunford, G. Coupland, and D. A. Laurie, "The evolution of CONSTANS-like gene families in barley, rice, and Arabidopsis," Plant Physiology, vol. 131, no. 4, pp. 1855-1867, 2003.

[33] A. Diallo, N. Kane, Z. Agharbaoui, M. Badawi, and F. Sarhan, "Heterologous expression of wheat VERNALIZATION 2 ( $\mathrm{Ta}$ VRN2) gene in Arabidopsis delays flowering and enhances freezing tolerance," PloS One, vol. 5, no. 1, Article ID e8690, 2010.

[34] N. A. Kane, J. Danyluk, G. Tardif et al., “TaVRT-2, a member of the StMADS-11 clade of flowering repressors, is regulated by vernalization and photoperiod in wheat," Plant Physiology, vol. 138, no. 4, pp. 2354-2363, 2005.

[35] N. A. Kane, Z. Agharbaoui, A. O. Diallo et al., "TaVRT2 represses transcription of the wheat vernalization gene TaVRN1," Plant Journal, vol. 51, no. 4, pp. 670-680, 2007.

[36] S. D. Michaels, G. Ditta, C. Gustafson-Brown, S. Pelaz, M. Yanofsky, and R. M. Amasino, "AGL24 acts as a promoter of flowering in Arabidopsis and is positively regulated by vernalization," Plant Journal, vol. 33, no. 5, pp. 867-874, 2003. 
[37] A. Greenup, W. J. Peacock, E. S. Dennis, and B. Trevaskis, "The molecular biology of seasonal flowering-responses in Arabidopsis and the cereals," Annals of Botany, vol. 103, no. 8, pp. 1165-1172, 2009.

[38] D. Weigel and E. M. Meyerowitz, "The ABCs of floral homeotic genes," Cell, vol. 78, no. 2, pp. 203-209, 1994.

[39] J. Schmitz, R. Franzen, T. H. Ngyuen et al., "Cloning, mapping and expression analysis of barley MADS-box genes," Plant Molecular Biology, vol. 42, no. 6, pp. 899-913, 2000.

[40] L. Yan, A. Loukoianov, G. Tranquilli, M. Helguera, T. Fahima, and J. Dubcovsky, "Positional cloning of the wheat vernalization gene VRN1," Proceedings of the National Academy of Sciences of the United States of America, vol. 100, no. 10, pp. 6263-6268, 2003.

[41] K. Petersen, E. Kolmos, M. Folling et al., "Two MADS-box genes from perennial ryegrass are regulated by vernalization and involved in the floral transition," Physiologia Plantarum, vol. 126, no. 2, pp. 268-278, 2006.

[42] J. C. Preston and E. A. Kellogg, "Discrete developmental roles for temperate cereal grass VERNALIZATION 1/FRUITFULLike genes in flowering competency and the transition to flowering," Plant Physiology, vol. 146, no. 1, pp. 265-276, 2008.

[43] S. Sasani, M. N. Hemming, S. N. Oliver et al., "The influence of vernalization and daylength on expression of floweringtime genes in the shoot apex and leaves of barley (Hordeum vulgare)," Journal of Experimental Botany, vol. 60, no. 7, pp. 2169-2178, 2009.

[44] M. O. Winfield, C. Lu, I. D. Wilson, J. A. Coghill, and K. J. Edwards, "Cold- and light-induced changes in the transcriptome of wheat leading to phase transition from vegetative to reproductive growth," BMC Plant Biology, vol. 9, p. 55, 2009.

[45] F. Turck, F. Fornara, and G. Coupland, "Regulation and identity of florigen: flowering locus T moves center stage," Annual Review of Plant Biology, vol. 59, pp. 573-594, 2008.

[46] J. A. Zeevaart, "Leaf-produced floral signals," Current Opinion in Plant Biology, vol. 11, no. 5, pp. 541-547, 2008.

[47] M. D’Aloia, D. Bonhomme, F. Bouché et al., "Cytokinin promotes flowering of Arabidopsis via transcriptional activation of the FT paralogue TSF," Plant Journal, vol. 65, no. 6, pp. 972979, 2011.

[48] Y. He, M. R. Doyle, and R. M. Amasino, "PAF1-complexmediated histone methylation of Flowering Locus $C$ chromatin is required for the vernalization-responsive, winter-annual habit in Arabidopsis," Genes and Development, vol. 18, no. 22, pp. 2774-2784, 2004.

[49] S. Oh, H. Zhang, P. Ludwig, and S. Van Nocker, "A mechanism related to the yeast transcriptional regulator Paflc is required for expression of the Arabidopsis FLC/MAF MADS box gene family," Plant Cell, vol. 16, no. 11, pp. 2940-2953, 2004.

[50] S. Fujiwara, A. Oda, R. Yoshida et al., "Circadian clock proteins LHY and CCA1 regulate SVP protein accumulation to control flowering in Arabidopsis," Plant Cell, vol. 20, no. 11, pp. 29602971, 2008.

[51] G. G. Simpson, "The autonomous pathway: epigenetic and post-transcriptional gene regulation in the control of Arabidopsis flowering time," Current Opinion in Plant Biology, vol. 7 , no. 5, pp. 570-574, 2004. 

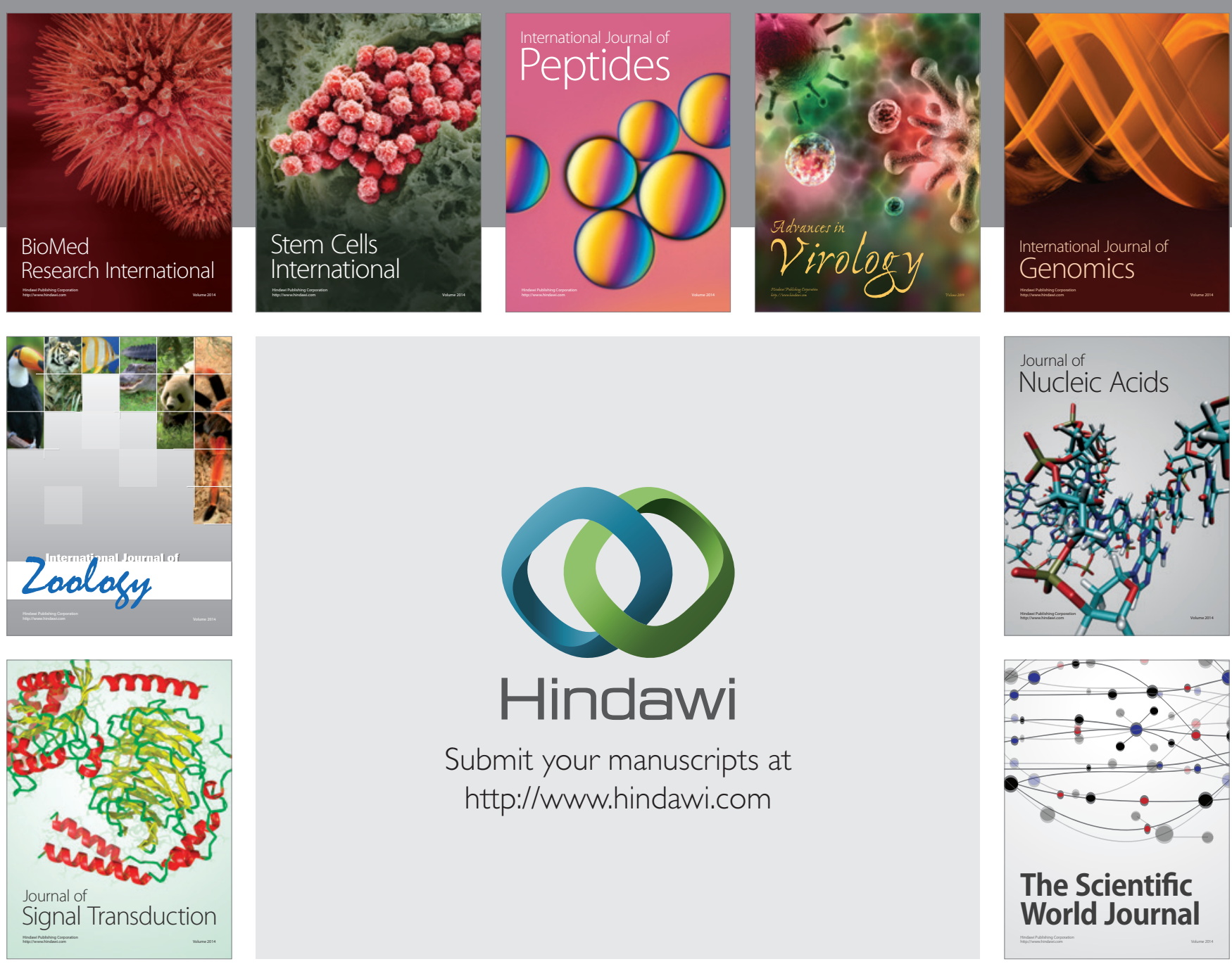

Submit your manuscripts at

http://www.hindawi.com
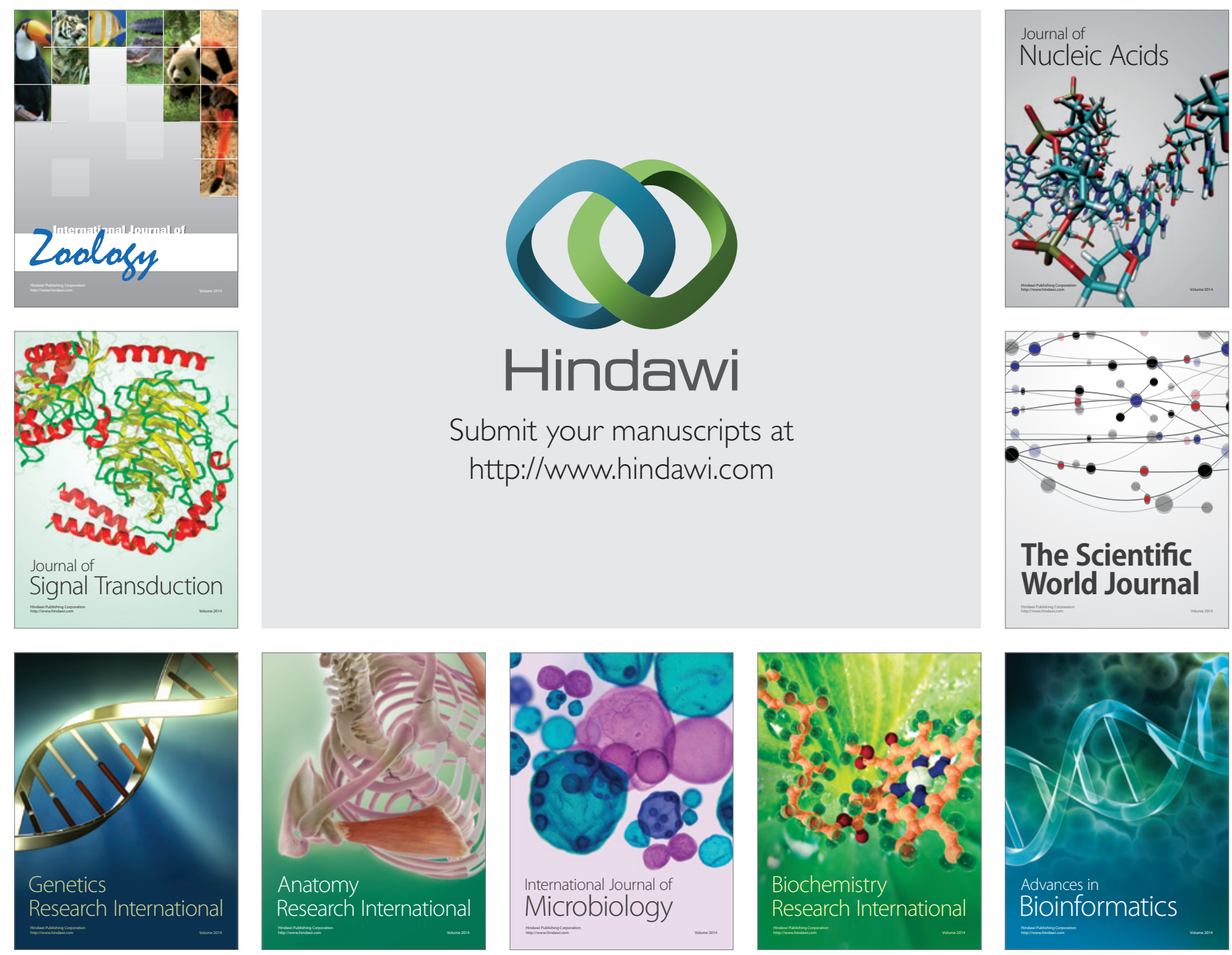

The Scientific World Journal
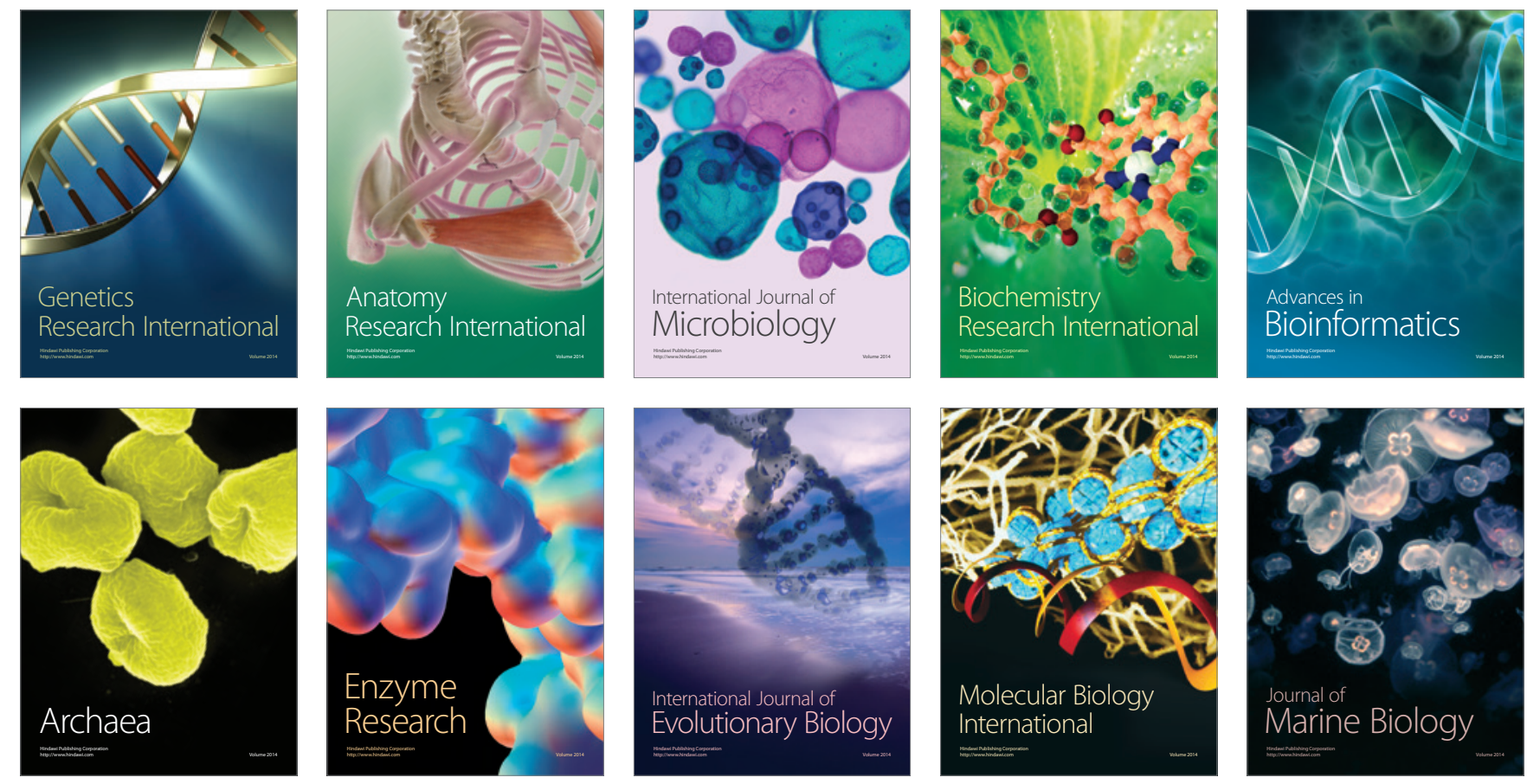